\title{
The Quantum Theory of Second Class Constraints: Kinematics
}

\author{
Hendrik Grundling ${ }^{1}$ and C. A. Hurst ${ }^{2}$ \\ ${ }^{1}$ Department of Mathematics, I.A.S., Australian National University, GPO Box 4, Canberra, ACT \\ 2601, Australia \\ ${ }^{2}$ Department of Physics and Mathematical Physics, University of Adelaide, GPO Box 498, Adelaide, \\ SA 5001 Australia
}

\begin{abstract}
The problem of second class quantum constraints is here set up in the context of $C^{*}$-algebras, utilizing the connection with state conditions as given by the heuristic quantization rules. That is, a constraint set is said to be first class if all its members can satisfy the same state condition, and second class otherwise. Several heuristic models are examined, and they all agree with this definition. Given then a second class constraint set, we separate out its first class part as all those constraints which are compatible with the others, and we propose an algebraic construction for imposition of the constraints. This construction reduces to the normal one when the constraints are first class. Moreover, the physical automorphisms (assumed as conserving the constraints) will also respect this construction. The final physical algebra obtained is free of constraints, gauge invariant, unital, and with the right choice, simple. This $C^{*}$-algebra also contains a factor algebra of the usual observables, i.e. the commutator algebra of the constraints. The general theory is applied to two examples - the elimination of a canonical pair from a boson field theory, as in the two dimensional anomalous chiral Schwinger model of Rajaraman [14], and the imposition of quadratic second class constraints on a linear boson field theory.
\end{abstract}

\section{Introduction}

The classical treatment of degenerate systems by the Dirac procedure [1] has reached a high degree of mathematical maturity in the symplectic formulation of Gotay, Nester and Hinds [2]. As for the quantum setting, these systems still remain within heuristic formulations [3] without drawing much from their classical rigor, due to the dubious nature of quantization [4]; geometric quantization [5] as yet being only a prequantization. It is our aim here and in our previous papers [6-8] to give a quantum mechanical procedure for eliminating degeneracy in a mathematically consistent way, and we do this in a $C^{*}$-algebra framework. 
The usual stated intention for applying the Dirac-Bergmann algorithm, is to prepare the classical theory in a suitable Hamiltonian form for quantization, the latter process is then implemented by an altered set of heuristic rules [3]. Now since only linear theories can be consistently quantized, we expect that the Dirac procedure will not in general solve the mathematical difficulties of quantum degenerate systems. For instance, it is meant to remove all second class constraints so that these do not enter the theory at all. However, second class constraints can easily arise in the quantum framework, cf. [17] since there are quantum degeneracies without classical counterparts, for which one may wish to impose supplementary conditions. Indeed, it is necessary to define more precisely what we mean by first and second class variables in the quantum picture. It therefore appears that the problem of degeneracy must be solved afresh for the quantum setting. Nevertheless, we would do well to remain close to the structures of the Dirac procedure, considering its proven utility in heuristic physics. Hence we recall the Dirac algorithm in its simplest form. The theoretical motivation behind it can be found in any one of a number of well known texts [1].

(1) First obtain the set of primary constraints $\left\{\Omega_{i}\right\}$ as relations between canonical variables.

(2) Add a combination of these to the Hamiltonian: $H_{P}:=H+\alpha_{i} \Omega_{i}$.

(3) Put the time evolutions of $\left\{\Omega_{i}\right\}$ under $H_{P}$ weakly to zero to obtain the set of secondary constraints. Fix the relevant multipliers $\alpha_{i}$ to minimize this set. Continue this process with the augmented constraint set until it terminates. Only the second class constraints have their multipliers fixed.

(4) Separate the full constraint set $\left\{\Gamma_{i}\right\}$ into first-class $\left\{\chi_{i}\right\}$ and second-class $\left\{\xi_{i}\right\}$ sets according to whether their Poisson brackets with all the other constraints weakly vanish or not. Define the observables as the first class phase space functionals.

(5) Construct the Dirac bracket $\{\cdot, \cdot\}_{D}$ using the second class constraints $\left\{\xi_{i}\right\}$.

(6) Let $\{\cdot, \cdot\}_{D}$ define a new Lie algebra on the phase space functionals, hence eliminate the second class constraints.

(7) Quantize heuristically with the rule $\{\cdot, \cdot\}_{D} \rightarrow i[\cdot, \cdot]$ and by selecting the physical states by the condition $\chi_{i}|\psi\rangle=0$.

The reason why the second class constraints are not allowed into the quantum theory is because they would give rise to inconsistencies if one attempts to impose them in the quantum theory by $\xi_{i}|\psi\rangle=0$. A good example of this is a canonical pair $q, p$; if $q|\psi\rangle=p|\psi\rangle=0$, then clearly $\langle\psi|[q, p]| \psi\rangle=0$ contradicts $[q, p]=i$. In fact, Shanmugadhasan [9] showed that any classical degenerate system can be canonically transformed into a form in which its second class constraints are all expressed as canonical pairs, hence in the following a canonical pair will be a good example of second class constraints against which we can test our theory. Now in a quantum theory there may be many alternative methods apart from state conditions for eliminating constraints, and we examine some below. To retain contact with the heuristic theory, we will define a set of constraints as first class in the quantum theory if its elements can be simultaneously annihilated by a state 
condition, and consider it as second class otherwise. The straightforward adaptation of the classical definition does not work. We have already worked out the algebraic theory associated with state conditions [6], and will employ that theory here.

The plan of this article is as follows. In Sect. 2 we recall for reference the salient points of the $T$-procedure for state conditions, together with an application to linear boson fields and external constraints. Section 3 sets up the kinematics problem of second class constraints and looks at a few typical second class situations. In Sect. 4 we separate out the first class constraints from the full set, and in Sect. 5 we impose the constraints to obtain the final physical algebra, having first considered a construction suggested by analogy with the Dirac procedure. Through all this, we show that the chosen constructions are preserved by physical transformations. Section 6 consists of applications of the general theory, containing the elimination of a canonical pair and imposition of quadratic constraints, and in Sect. 7 we discuss the problem of dynamics adjustment.

\section{The $T$-Procedure: Structures Enforced by State Conditions}

Given a hermitian heuristic condition $A|\psi\rangle=0$ which implies $\langle\psi|\exp i \lambda A| \psi\rangle=1$, we prefer to work with the latter in order to avoid problems with unbounded operators. In the rigorous theory we therefore assume a distinguished set of unitaries $U$ which is connected to $\exp i \lambda A$ under some correspondence rule.

In this section we collect the basic algebraic structures associated with systems with state conditions, as developed in [6-8], which is where the interested reader can find the proofs of the statements below. As in [10], assume:

2.1. All physical information of a specified system is contained in the pair $\mathscr{F}, \mathfrak{\Xi}$, where the unital $C^{*}$-algebra $\mathscr{F}$ is the field algebra, and $\mathfrak{G}$ is its set of states.

The degeneracy assumption is:

2.2. There is a specified unitary set $\mathscr{U}$ in $\mathscr{F}$ called state conditions. All physical information is contained in $\mathscr{F}$ and the set of Dirac states defined by:

$$
\mathfrak{S}_{D}:=\{\omega \in \mathfrak{S} \mid\langle\omega \mid U\rangle=1 \forall U \in \mathscr{U}\} \text {. }
$$

Then $\omega \in \Xi_{D}$ iff $\langle\omega \mid A U\rangle=\langle\omega \mid A\rangle=\langle\omega \mid U A\rangle \forall U \in \mathscr{U}, \forall A \in \mathscr{F}$, (hence we can extend the state conditions $\mathscr{U}$ to the group generated by it) or in terms of $L(U):=U-\mathbb{1}: \omega \in \mathbb{S}_{D}$ iff $\{L(\mathscr{U})\} \subset \operatorname{Ker} \omega$ iff $\mathscr{F}\{L(\mathscr{U})\} \cup\{L(\mathscr{U})\} \mathscr{F} \subset \operatorname{Ker} \omega$. If for each $U \in \mathscr{U}$ we define the automorphism $\alpha_{U}:=\operatorname{Ad} U$, then it is clear from the above that the Dirac states are all $\mathscr{U}$-invariant, $\mathfrak{\Xi}_{D} \subset \mathfrak{\subseteq}^{\mathscr{U}}$, i.e. $\left\langle\omega \mid \alpha_{U}(A)\right\rangle=\langle\omega \mid A\rangle \forall U \in \mathscr{U}$, $A \in \mathscr{F}, \omega \in \mathfrak{\Xi}_{D}$.

Theorem 2.3. Let $\mathscr{A}(L)$ be the $C^{*}$-algebra generated by $\{L(\mathscr{U})\}$. Then $\omega \in \Theta_{D}$ iff $\mathscr{A}(L) \subset \operatorname{Ker} \omega$ iff $[\mathscr{A}(L) \mathscr{F} \cup \mathscr{F} \mathscr{A}(L)] \subset \operatorname{Ker} \omega$, where $[\cdot]$ denotes the closed linear space generated by its argument.

Theorem 2.4. $\mathfrak{S}_{D} \neq \emptyset$ iff $\mathbb{1} \notin \mathscr{A}(L)$ iff $\mathbb{1} \notin[\mathscr{A}(L) \mathscr{F} \cup \mathscr{F} \mathscr{A}(L)]$, and in this case $\mathfrak{S}_{D}$ contains pure states.

So our nontriviality assumption is: 
2.5. Henceforth assume $1 \notin \mathscr{A}(L)$.

We also denote $\mathscr{A}(L)$ by $C^{*}(\mathscr{U}-1)$. For any set $\Omega \subset \mathscr{F}$, define:

$$
\mathscr{M}_{\mathscr{F}}(\Omega):=\{F \in \mathscr{F} \mid F M \in \Omega \ni M F \forall M \in \Omega\} .
$$

hence if $\Omega$ is a $C^{*}$-algebra, then $\mathscr{M}_{\mathscr{F}}(\Omega)$ is the largest $C^{*}$-algebra in $\mathscr{F}$ for which $\Omega$ is a two sided ideal.

Theorem 2.6. Let $\mathscr{N}:=[\mathscr{F} \mathscr{A}(L)], \mathscr{D}:=\mathscr{N} \cap \mathscr{N}^{*}$, then $\mathscr{D}$ is the largest $C^{*}$-algebra annihilated by all the Dirac states, i.e. $\mathscr{D}$ is the unique maximal $C^{*}$-algebra in $\mathscr{K}:=\cap\left\{\operatorname{Ker} \omega \mid \omega \in \mathbb{S}_{D}\right\}$.

Theorem 2.7. $\mathcal{O}:=\{F \in \mathscr{F} \mid[F, H] \in \mathscr{D} \forall H \in \mathscr{D}\}=\mathscr{M}_{\mathscr{F}}(\mathscr{D})$.

Then $1 \notin \mathscr{D}$, and $\mathscr{D}$ is a proper two-sided ideal for $\mathcal{O}$. In [1], Dirac defines his observables as "first-class variables" in an analogous way to the way that $\mathcal{O}$ is here defined, i.e. as the "weak commutant" of the constraints. The observables in quantum theories are traditionally taken to be $\mathscr{A}(L)^{\prime}$.

Define $\mathscr{S}$ to be the largest set such that $\mathscr{A}(L) \mathscr{S} \subset[\mathscr{F} \mathscr{A}(L)]$. Then $1 \in \mathscr{A}(L)^{\prime} \subset$ $\mathscr{S} \cap \mathscr{S}^{*}$.

Theorem 2.8. $\mathscr{D}=\overline{\mathscr{S} * \mathscr{A}(L) \mathscr{S}}$ and $\mathscr{O}=\mathscr{S} \cap \mathscr{S}^{*}$.

Hence $\mathscr{A}(L)^{\prime} \subset \mathcal{O}$, and so we could choose $\mathcal{O}$ even as the set of observable quantities. $\mathcal{O}$ can be considered as the largest $C^{*}$-algebra on which we can consistently impose the constraints. Define the maximal $C^{*}$-algebra of physical observables as

$$
\mathscr{R}:=\mathcal{O} / \mathscr{D} \text {. }
$$

The factoring procedure is the actual step of imposing the constraints. Now it is possible that $\mathscr{R}$ may not be simple, and this would not be acceptable for a physical algebra. So, using the physical arguments, one would in practice choose a $C^{*}$-subalgebra $\mathcal{O}_{c} \subseteq \mathcal{O}$ containing $\mathscr{A}(L)^{\prime}$ such that

$$
\mathscr{R}_{c}:=\mathcal{O}_{c} /\left(\mathscr{D} \cap \mathcal{O}_{c}\right) \subset \mathscr{R}
$$

is simple, and then $\mathscr{R}_{c}$ is the right physical algebra. The distinction between $\mathcal{O}$ and $\mathcal{O}_{c}$ was not made in [6]. We call the procedure for obtaining the objects above the $T$-procedure. $\mathcal{O}$ consists of the "weakly gauge invariant elements."

Theorem 2.9. $A \in \mathcal{O}$ iff $\alpha_{U}(A)-A \in \mathscr{D} \forall U \in \mathscr{U}$, i.e. $\left(\alpha_{\mathscr{U}}-\imath\right) A \subset \mathscr{D}$.

Theorem 2.10. $\omega \in \subseteq_{D}$ iff $\pi_{\omega}(\mathscr{D}) \Omega_{\omega}=0$, where $\pi_{\omega}$ and $\Omega_{\omega}$ are respectively the GNS-representation of $\omega$ and its cyclic vector.

This corresponds to the heuristic $\chi|\psi\rangle=0$ method for imposing constraints. Define $Y:=\{\alpha \in$ Aut $\mathscr{F} \mid \mathscr{D}=\alpha[\mathscr{D}]\}$, then since $\mathcal{O}=\mathscr{M}_{\mathscr{F}}(\mathscr{D}), \alpha$ also preserves $\mathcal{O}$ and so defines canonically an automorphism $\alpha^{\prime}$ on $\mathscr{R}$. Define the group homomorphism $T: \Upsilon \mapsto$ Aut $\mathscr{R}$ by $T(\alpha)=\alpha^{\prime}$, then we expect $\operatorname{Ker} T$ to consist of gauge transformations:

Theorem 2.11. Ker $T=\left\{\alpha \in \operatorname{Aut} \mathscr{F} \mid\langle\omega \mid \alpha[A] F\rangle=\langle\omega \mid A F\rangle \forall A, F \in \mathcal{O}\right.$ and $\left.\forall \omega \in \mathbb{S}_{D}\right\} \subset Y$.

Theorem 2.12. $\alpha \in \operatorname{Inn} \mathscr{F} \cap \Upsilon \Rightarrow \alpha^{\prime} \in \operatorname{Inn} \mathscr{R}$.

The physically admissible automorphisms of $\mathscr{F}$ denoted by $Y_{c}$ are those which 
are definable on $\mathscr{R}_{c}$, i.e. $\alpha\left(\mathcal{O}_{c}\right)=\mathcal{O}_{c}$, and $\alpha\left(\mathscr{D} \cap \mathcal{O}_{c}\right) \subseteq \mathscr{D} \cap \mathscr{O}_{c}$. Clearly, if $\alpha \in \Upsilon$, it is sufficient that it satisfies $\alpha\left(\mathcal{O}_{c}\right) \subseteq \mathcal{O}_{c}$ for it to be physically admissible.

For later reference we sketch the structures found for a linear boson field with linear hermitian constraints, such as the Dirac form of electromagnetism, cf. [6]. The field algebra is taken as Manuceau's $C^{*}$-algebra of the CCR cf. $[10,11]$ : $\mathscr{F}=\overline{\Delta(\mathscr{M}, B)}$, where $\mathscr{M}$ is the complexified test function space with the nondegenerate symplectic form $B(\cdot, \cdot)$ on it. To fix notation for the later sections, we define $\overline{\Delta(\mathscr{M}, B)}$.

Definition 2.13. i) Given a linear topological space $\mathscr{M}$ with symplectic form $B$ on it, let $\Delta(\mathscr{M}, B)$ be the normed *-algebra such that its elements are the complex valued functions on $\mathscr{M}$ with finite support. It has the obvious linear structure, the following multiplication law:

$$
\left(f_{1} f_{2}\right)(z):=\sum_{z_{1} \in \mathscr{M}} f_{1}\left(z_{1}\right) f_{2}\left(z-z_{1}\right) \exp \left[-i B\left(z_{1}, z\right) / 2\right]
$$

involution $f^{*}(z):=\overline{f(-z)}$ and the norm $\|f\|_{1}:=\sum_{z \in \mathscr{M}}|f(z)|$.

ii) Denote the completion of $\Delta(\mathscr{M}, B)$ in the latter norm by $\Delta_{1}(\mathscr{M}, B)$. Then $\overline{\Delta(\mathscr{M}, B)}$ is the enveloping $C^{*}$-algebra of $\Delta_{1}(\mathscr{M}, B)$, i.e. the closure in the enveloping $C^{*}$-norm: $\|f\|:=\sup _{\pi \in P}\|\pi(f)\|$, where $P$ is the set of nondegenerate representations of $\Delta_{1}(\mathscr{M}, B)$.

The $C^{*}$-norm defined here appears to be different from the one defined by Manuceau, but by Slawny's uniqueness theorem [16], they are actually equal. The functions $\delta_{F}$ of support $\{F\}$ and value 1 form a generating set of unitaries for $\mathscr{F}$. The heuristic correspondence rule is $\delta_{F} \leftrightarrow W(F)$, where $W(F)$ is the heuristic Weyl operator constructed from the smeared version of a set of canonical pairs $p_{i}(x), q_{i}(x)$. The constraint group $\mathscr{U}$ is then specified as the group generated by $\delta_{C}:=\left\{\delta_{F} \mid F \in \mathscr{C}\right\}$, where $\mathscr{C}$ is a real linear subspace of $\mathscr{M}$ corresponding to the heuristic constraints. With

$$
\mathbf{p}:=\{H \in \mathscr{M} \mid B(H, C)=0 \forall C \in \mathscr{C}\},
$$

we found that $\delta_{\mathrm{p}}=\delta_{\mathscr{M}} \cap \mathcal{O}$, and indeed $C^{*}\left(\delta_{\mathrm{p}}\right)=C^{*}\left(\delta_{\mathscr{M}} \cap \mathcal{O}\right)=\mathscr{A}(L)^{\prime}$. There are additional elements to these in $\mathcal{O}$, of the form $\sum_{i} \alpha_{i} \delta_{F_{t}}$ with $F_{i} \notin \mathbf{p} \forall i$, but it is very difficult to get our hands on these, and so we make the choice: $\mathcal{O}_{c}=C^{*}\left(\delta_{\mathrm{p}}\right)=\mathscr{A}(L)^{\prime}$. Now $\mathscr{A}(L) \subset \mathcal{O}_{c}$, i.e. $\mathscr{C} \subset \mathbf{p}$, (because otherwise $\mathbb{1} \in \mathscr{A}(L)$ ), and so $\mathscr{A}(L)$ is commutative, and $\mathscr{U}=\delta_{\mathscr{b}}$. Then $\mathscr{D} \cap \mathscr{U}_{c}=\mathscr{A}(L) C^{*}\left(\delta_{\mathrm{p}}\right)$, and the physical algebra is $\mathscr{R}_{c}=$ $\mathscr{A}(L) / \mathscr{A}(L)^{\prime} \mathscr{A}(L)$. When $\mathscr{C}$ is the degenerate part of $\mathbf{p}$ with respect to $B$, then $\mathscr{R}_{c}=\overline{\Delta(\mathbf{p} / \mathscr{C}, \widetilde{B})}$, where $\widetilde{B}$ is the canonical image of $B$ on $\mathbf{p} / \mathscr{C}$ (which is nondegenerate), and so in this case $\mathscr{R}_{c}$ is simple. The symplectic transformations on $\mathscr{M}$ which conserve $\mathscr{C}$ will define automorphisms on $\mathscr{R}_{c}$. The states on $\mathscr{R}_{c}$ which will define physically relevant representations, (i.e. for a representation $\pi$ the map $\lambda \mapsto \pi\left(\delta_{\lambda_{F}}\right)$ for fixed $F$ is strongly continuous) are those for which the function $\lambda \mapsto \omega\left(\delta_{\lambda F}\right)$ is continuous for fixed $F \in \mathbf{p} / \mathscr{C}$.

In [7] we exhibited the connection between the structures above, and the usual 
structures of indefinite inner product representations, but we omit a discussion of that here, since it will not be utilized.

Finally, we give the structures of the situation when the constraints can only be defined as automorphisms on the field algebra, and the physical states are selected by invariance under these automorphisms [8]. This is the case when for instance quadratic constraints are imposed on a linear field algebra. The assumption is:

2.14. Given the situation of 2.1 , there is a distinguished group action $\alpha: G \mapsto$ Aut $\mathscr{F}$ on $\mathscr{F}$, and all physical information is contained in $\mathscr{F}$ and its set of invariant states:

$$
\mathfrak{S}^{G}(\mathscr{F}):=\left\{\omega \in \mathfrak{S}(\mathscr{F}) \mid \omega\left(\alpha_{g}(A)\right)=\omega(A) \forall g \in G, A \in \mathscr{F}\right\} .
$$

If $G$ is locally compact, we can construct $\mathscr{F}_{e}=M\left(G_{\alpha}^{\times} \mathscr{\mathscr { F }}\right)$ and if $G$ is an inductive limit of locally compact groups, $G=\lim _{\rightarrow} G_{i}$, we can form $\mathscr{F}_{e}=\lim _{\rightarrow} C^{*}\left(U_{G_{t}} \cup \widetilde{F}\right)$, where $U_{G_{l}}$ are the elements in $M\left(G_{l} \overrightarrow{\times} \overrightarrow{\mathscr{F}}\right)$ which implement $\alpha_{G_{l}}, \overrightarrow{\text { i.e. }} \alpha_{q}=\operatorname{Ad} U_{g}$ $\forall g \in G_{i}$. So in either case, we obtain a $C^{*}$-algebra $\mathscr{F}_{e} \supset \mathscr{F}$ which contains unitaries $U_{g} \forall g \in G$ that implement $\alpha: G \mapsto$ Aut $\mathscr{F}$. Then this situation is reduced to the previous one by the following theorem:

Theorem 2.15. $\mathfrak{G}^{G}(\mathscr{F})$ is precisely the restriction to $\mathscr{F}$ of the Dirac states on $\mathscr{F}_{e}$ with respect to $U_{G}$, i.e. $\mathfrak{S}^{G}(\mathscr{F})=\mathfrak{S}_{D}\left(\mathscr{F}_{e}\right) \mid \mathscr{F}$, where

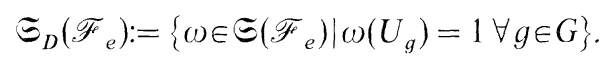

Hence we can apply the $T$-procedure to $U_{G}$ in $\mathscr{F}_{e}$, and intersect the resulting algebraic structures with $\mathscr{F}$. That is, $\mathscr{A}(L)=C^{*}\left(U_{G}-1\right), \mathscr{D}_{e}=\mathscr{A}(L) \mathscr{F}_{e} \cap \mathscr{F}_{e} \mathscr{A}(L)$,

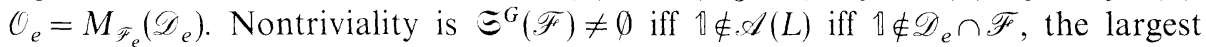
$C^{*}$-algebra in $\cap\left\{\operatorname{Ker} \omega \mid \omega \in \mathbb{\Xi}^{G}(\mathscr{F})\right\}$ is $\mathscr{D}_{e} \cap \mathscr{F}$, and the maximal physical algebra which is gauge invariant and constraint free, is $\mathscr{R}=\left(\mathcal{O}_{e} \cap \mathscr{F}\right) /\left(\mathscr{D}_{e} \cap \mathscr{F}\right)$. Physical automorphisms on $\mathscr{F}$ are those definable on $\mathscr{R}$. Clearly the gauge invariant algebra $\mathscr{F}^{G}$ is contained in $\mathcal{O}_{e} \cap \mathscr{F}$.

\section{Setting up the Problem}

The setting for the kinematics problem of the second class constraints problem is as follows:

3.0. Assume there is given a unital $C^{*}$-algebra $\mathscr{F}$ as a field algebra, and its set of states $\mathfrak{\Xi}$. Within $\mathscr{F}$ the constraints are specified as a set of unitaries $\mathscr{U} \subset \mathscr{F}_{U}$, and a set of physical automorphisms $\mathscr{E} \subset$ Aut $\mathscr{F}$ is given such that $\alpha(\mathscr{U}) \subset \mathscr{U} \forall \alpha \in \mathscr{E}$. The physical structures should be such that $\mathscr{U}$ acts trivially on these.

The omission of the nontriviality assumption 2.5 , means that we cannot assume that $\mathscr{U}$ can be set to $\mathbb{1}$ by state conditions. Moreover, the assumption that $\mathscr{E}$ preserves $\mathscr{U}$ means that we start at step 4 of the Dirac procedure, taking the dynamics problem, i.e. the adjustment of the dynamics in order to preserve $\mathscr{U}$, as solved. This problem is discussed in Sect. 7. As before, $\mathscr{U}$ corresponds to the heuristic constraints $\left\{\Gamma_{i}\right\}$ according to $\mathscr{U} \leftrightarrow\left\{\exp i \lambda \Gamma_{i}\right\}$.

In this work, it is our aim to solve the following problem. Obtain from $\mathscr{F}$ a $C^{*}$-algebra $\mathscr{R}$ which is unital, constraint free, gauge invariant and containing the 
traditional observables $\mathscr{U}^{\prime}$ (modulo the first class constraints). $\mathscr{E}$ should also be definable on $\mathscr{R}$, and when $1 \notin C^{*}(\mathscr{U}-\mathbb{1}), \mathscr{R}$ should coincide with the physical algebra $\mathscr{R}$ obtained through application of the $T$-procedure to $\mathscr{U}$.

We now need to define what is meant by first and second class constraints in the quantum setting. Dirac defined a constraint $\chi_{i}$ as first class if the Poisson bracket $\left\{\chi_{i}, \Gamma_{i}\right\}_{P B}$ is a linear combination of constraints, where $\Gamma_{i}$ ranges over the full set of constraints. The coefficients of this combination are phase space functionals. We found that all direct adaptations of this definition to the present framework failed.

Now recall that in step 7 of the Dirac algorithm, one requires that the quantized first class constraints annihilate the physical vectors, i.e. they should all satisfy simultaneously a state condition. This is what we will take as our definition of first class constraints. By the nontriviality condition 2.4 , we see that a constraint set $\mathscr{U} \subset \mathscr{F}_{L}$ will satisfy a state condition iff $1 \notin \mathscr{A}(L)=C^{*}(\mathscr{U}-\mathbb{1})$.

Definition 3.1. We say a set of unitaries $\mathscr{U} \subset \mathscr{F}_{U}$ is first class if $1 \notin C^{*}(\mathscr{U}-\mathbb{1})$, and if $\mathbb{1} \in C^{*}(\mathscr{U}-\mathbb{1})$, i.e. $C^{*}(\mathscr{U})=C^{*}(\mathscr{U}-\mathbb{1})$, we say $\mathscr{U}$ contains second class constraints.

Hence the situation set up in 3.0 admits second class constraints. Let $L_{i}:=U_{i}-1$, $U_{i} \in \mathscr{U}$. Then $L_{1} L_{2}+L_{1}+L_{2}=U_{1} U_{2}-\mathbb{1} \in C^{*}(\mathscr{U}-1) \subset \mathscr{D}$. Hence, given a first class set $\mathscr{U}$, the group generated by it $\langle\mathscr{U}\rangle$ will also select the same set of physical states $\Xi_{D}$, and the same physical algebra $\mathscr{R}$, and $\omega(\mathscr{U})=1=\omega(\langle\mathscr{U}\rangle) \forall \omega \in \Xi_{D}$, i.e. the Dirac states "respect" the group structure of $\langle\mathscr{X}\rangle$. Now the states are also linear functionals, hence we expect that if a set $\mathscr{U}$ contains second class constraints, then the group structure of $\langle\mathscr{U}\rangle$ will clash with its linear structure in $\mathscr{F}$ with respect to the Dirac states. This is what the next lemma says.

Lemma 3.2. Given a set $\mathscr{U} \subset \mathscr{F}_{U}$, we have that $\mathbb{1} \in C^{*}(\mathscr{U}-\mathbb{1})$ iff $\exists U \in\langle\mathscr{U}\rangle$ such that $U=\sum_{l} \lambda_{l} U_{l}$, where $U_{l} \in\langle\mathscr{U}\rangle$, and $\sum_{l} \lambda_{l} \neq 1$.

Proof. Clearly if $\sum \lambda_{l} U_{l} \in\langle\mathscr{U}\rangle \ni U_{l}, \sum \lambda_{l} \neq 1$, there are no states $\omega$ satisfying $\omega(\mathscr{U})=\omega(\langle\mathscr{U}\rangle)=1$ because $\omega\left(\sum \lambda_{l} U_{l}\right)=\sum \lambda_{l} \neq 1$ is a contradiction. Hence $\Xi_{D}=\emptyset$, and so by 2.4, $\mathbb{1} \in C^{*}(\mathscr{U}-\mathbb{1})$. Conversely, assume that for all $U \in\langle\mathscr{U}\rangle$ of the form $U=\sum \lambda_{l} U_{l}, U_{l} \in\langle\mathscr{U}\rangle$, that $\sum \lambda_{l}=1$. Now $C^{*}(\mathscr{U})$ is the closed linear space generated by $\langle\mathscr{U}\rangle$, so a state on $C^{*}(\mathscr{U})$ is uniquely specified by its values on $\langle\mathscr{U}\rangle$. Hence choose a linear independent set $\left\{U_{\alpha}\right\} \subset\langle\mathscr{U}\rangle$, then any linear functional on $C^{*}(\mathscr{U})$ can be specified by arbitrarily choosing its values on $\left\{U_{\alpha}\right\}$. Specify $\omega\left(U_{\alpha}\right)=1 \forall \alpha$. Then by the assumption we get that $\sum \lambda_{\alpha}=1$ for all linear combinations $\sum \lambda_{\alpha} U_{\alpha} \in\langle\mathscr{U}\rangle$, and hence $\omega(\mathscr{U})=1$. We need to check that the specification $\omega\left(U_{\alpha}\right)=1$ defines a state, i.e. that $\omega\left(C^{*}(\mathscr{U})_{+}\right) \geqq 0$. For any $B^{*} B \in C^{*}(\mathscr{U})_{+}$with $B=\sum \hat{\lambda}_{\alpha} U_{\alpha} \in C^{*}(\mathscr{U})$, we get:

$$
\omega\left(B^{*} B\right)=\omega\left(\sum_{\alpha, \beta} \bar{\lambda}_{\alpha} \lambda_{\beta} U_{\alpha}^{-1} U_{\beta}\right)=\sum_{\alpha, \beta} \bar{\lambda}_{\alpha} \lambda_{\beta}=\left|\sum \lambda_{\alpha}\right|^{2} \geqq 0 .
$$

For the closure, limits are easily done. Hence $\omega$ is a state on $C^{*}(\mathscr{U})$, and can be extended by the Hahn-Banach theorem to a state on $\mathscr{F}$, and so $\omega \in \Xi_{D} \neq \emptyset$, i.e. $1 \notin C^{*}(\mathscr{U}-1)$. 
We now test Definition 3.1 against two simple examples where we know heuristically that $\mathscr{U}$ contains second class constraints.

First consider the heuristic second class situation where we want to put both $\xi$ and $\xi+\lambda 1$ equal to zero. In the present framework, this means $\mathscr{U}=\left\{U, e^{i \theta} U\right\}$, where $\theta \in \mathbb{R} \backslash 2 \pi \mathbf{Z}$. We immediately see from Lemma 3.2 that $\mathbb{1} \in C^{*}(\mathscr{U}-\mathbb{1})$, but we show this directly. With $L(U):=U-1$, we get $L(U)-L\left(e^{i \theta} U\right)=$ $\left(1-e^{i \theta}\right) U \in C^{*}(\mathscr{U}-1)$, hence $U \in C^{*}(\mathscr{U}-1)$, and so $C^{*}(\mathscr{U}-1)$ has unitaries, i.c. is unital, and so $\mathscr{U}$ is second class.

Next assume the situation of a canonical pair, i.e. $\mathscr{U}=\left\{\delta_{F}, \delta_{G}\right\}$, where as in Sect. 2 for the linear boson field, these satisfy

$$
\delta_{F} \cdot \delta_{G}=\delta_{F+G} \exp \frac{-i}{2} B(F, G),
$$

$B(F, G) \neq 0$. Then $\delta_{-G} \delta_{F} \delta_{G}=\delta_{F} \exp i B(F, G) \in\langle\mathscr{U}\rangle \ni \delta_{F}$, and so by Lemma 3.2, $C^{*}(\mathscr{U}-\mathbb{1})$ is unital, i.e. $\mathscr{U}$ is second class.

From these examples, we see that it would not be advisable to take $\langle\mathscr{U}\rangle$ as our constraint set instead of $\mathscr{U}$ when $\mathscr{U}$ has second class constraints. This is because in these examples, the group structure of $\langle\mathscr{U}\rangle$ will give every element a multiple of itself, and so the elements which may have been first class with respect to each of the other elements in $\mathscr{U}$ (a notion defined below), will become second class in $\langle\mathscr{U}\rangle$.

\section{Separating out the First Class Constraints}

In step 4 of the Dirac procedure, the constraints are separated into first and second class sets, which is what we intend to do here in the quantum framework. For convenience, let $\mathscr{U}$ be indexed by a set $I$ (which may be $\mathscr{U}$ itself), i.e. $\mathscr{U}=\left\{U_{j}\right\}_{j \in I} \subset \widetilde{\mathscr{F}}_{U}$ and for $R \subseteq I$, we denote $U_{R}:=\left\{U_{j} \mid j \in R\right\}$.

Definition 4.1. Call a set $R \subset I$ compatible if $U_{R}$ is first class, i.e. $\mathbb{1} \notin C^{*}\left(U_{R}-\mathbb{1}\right)$. Denote the set of compatible sets by $\mathscr{P}, \mathscr{P} \subset 2^{I}$. Then we say a set $P \subset I$ is compatible with a set $R \subset I$ if $P \cup R \in \mathscr{P}$ (and hence $P \in \mathscr{P} \ni R$ ).

Note that $\mathscr{P}$ with set inclusion becomes a partially ordered set, but it is not a lattice. We consider this definition as the quantum analog of Dirac's notion of constraints being compatible if they have weakly vanishing Poisson bracket. We can then form the following partition of $I, I=I_{i} \cup I_{r} \cup I_{0}$, where:

Definition 4.2. $I_{i}:=\left\{k \in I \mid\{k\} \notin \mathscr{P}\right.$, i.e. $\left.\mathbb{1} \in C^{*}\left(U_{k}-1\right)\right\}$, and we call these intrinsically second class. Call $I_{r}:=\{k \in I \mid \exists R \in \mathscr{P}$ such that $\{k\} \cup R \notin \mathscr{P} \ni\{k\}\}$ relatively second class; and then define the first class part of $I$ as $I_{0}:=\{k \in I \mid\{k\} \cup R \in \mathscr{P} \forall R \in \mathscr{P}\}$. Note that $k \in I_{0}$ implies that $\{k\} \in \mathscr{P}$.

The intrinsically second class elements $I_{i}$ are those which cannot be consistently set to 1 , e.g. $e^{i \theta} \mathbb{1}$ with $e^{i \theta} \neq 1$. We do not expect such elements to arise in reasonable theories. The relatively second class elements $I_{r}$ are those which are not compatible with some compatible set. A good example of the latter is a canonical pair $\delta_{F}, \delta_{G}, B(F, G) \neq 0$ as in the previous section. Finally, the first class part $I_{0}$ consists of all those elements which are compatible with all compatible sets. This nomenclature is in analogy with the Dirac algorithm, where the first class 
constraints are all those compatible (in Dirac's sense) with all the others. However, for $I_{0}$ to be acceptable in the quantum framework, we need to show that it is itself compatible, $I_{0} \in \mathscr{P}$.

Lemma 4.3. Let $\mathscr{A}$ be a $C^{*}$-algebra generated by a set $\left\{A_{i}\right\}_{i \in J} \subset \mathscr{A}$ (contained in a larger unital $C^{*}$-algebra) such that $\mathbb{1} \notin C^{*}\left(A_{F}\right)$ for all finite subsets $F \subset J$. Then $\mathbb{1} \notin \mathscr{A}$.

Proof. Note that $\mathscr{A}$ is an inductive limit of the $C^{*}$-algebras $C^{*}\left(A_{F}\right), F \subset J$, in the natural way. Then by Blackadar 3.3.1 [12], there is for any invertible $x \in \mathscr{A}$ a sufficiently large but finite set $F \in J$ such that $x \in C^{*}\left(A_{F}\right) \ni x^{-1}$. Hence given the hypothesis of the lemma, $1 \notin \mathscr{A}$.

Proposition 4.4. $\mathbb{1} \notin C^{*}\left(U_{I_{0}}-\mathbb{1}\right)$, i.e. $I_{0}$ is a compatible index set, $I_{0} \in \mathscr{P}$.

Proof. By 4.3 we only need to show $1 \notin C^{*}\left(U_{F}-1\right)$ for all finite subsets $F \subset I_{0}$. Build each $F$ up inductively. Each $k \in I_{0}$ is self-compatible, $\{k\} \in \mathscr{P}$. Let $R \subset I_{0} \cap \mathscr{P}$, and $k \in I_{0} \backslash R$. Then $k \cup C \in \mathscr{P} \forall C \in \mathscr{P}$ implies that $k \cup R \in \mathscr{P}$. Hence we can build up $F$ in a finite number of steps starting from a single element, and know at each step that it is a compatible set.

Recall that we also have a set of physical automorphisms $\mathscr{E}$ under which $\mathscr{U}=U_{I}$ is stable. Then for $U_{I_{0}}$ to be acceptable as the first class constraints, it too needs to be stable under $\mathscr{E}$.

Theorem 4.5. Given as above $\left\{U_{i}\right\}_{i \in I} \subset \mathscr{F} \ni 1$ and an $\alpha \in$ Aut $\mathscr{F}$ such that $\alpha\left(U_{I}\right)=U_{I}$, then $\alpha\left(U_{I_{0}}\right)=U_{I_{0}}$.

Proof. Since $\alpha(1)=1$, we see $1 \notin C^{*}\left(U_{R}-1\right)$ iff $1 \notin C^{*}\left(\alpha\left(U_{R}\right)-1\right) \forall R \in \mathscr{P}$, and similarly for $\alpha^{-1}$. Hence we can define a bijection $\alpha^{\prime}: \mathscr{P}_{\mapsto} \mathscr{P}$ by $\alpha\left(U_{R}\right)=: U_{\alpha^{\prime}(R)}$ since $\alpha\left(U_{I}\right)=U_{I}$. Now $k \in I_{0}$ iff $k \cup R \in \mathscr{P} \forall R \in \mathscr{P}$. Since $\alpha^{\prime}$ is a bijection, $\alpha^{\prime}(k) \cup R \in \mathscr{P}$ $\forall R \in \mathscr{P}$ iff $\alpha^{\prime}(k) \cup \alpha^{\prime}(R)=\alpha^{\prime}(k \cup R) \in \mathscr{P} \forall R \in \mathscr{P}$ iff $k \cup R \in \mathscr{P} \forall R \in \mathscr{P}$. Hence $\alpha^{\prime}(k) \in I_{0}$ iff $k \in I_{0}$, and so $\alpha\left(U_{I_{0}}\right)=U_{I_{0}}$.

To verify the physical reasonableness of Definitions 4.2 , one would also need to check it against some examples, i.e. whether the separation of the constraints into first and second class agrees with the heuristic results. This will be done in Sect. 6.

\section{Imposing the Constraints}

On having separated the first and second class constraints, we now get to step 5 of the Dirac algorithm, the construction of the Dirac bracket (DB). Since the linear field can be quantized consistently, we examine how the DB will affect it, in order to see how to adapt this concept to the quantum setting.

Observe first that the DB puts the second class constraints into the centre of the Lie algebra, and if we interpret the DB as a commutator in a $C^{*}$-algebra, then likewise it puts these constraints in the centre of the algebra but does not eliminate them. So in the quantum framework, we expect two parts to step 5 of the Dirac algorithm, the construction of a "quantum DB" and the elimination of the residual second class constraints in the centre. Moreover, on the observables (first-class 
elements), the DB should be weakly equal to the usual bracket.

Given a heuristic linear boson field with first and second class constraints denoted respectively $\left\{\chi_{i}\right\}$ and $\left\{\xi_{i}\right\}$, a canonical pair will not be canonical with respect to the DB any more:

$$
\begin{aligned}
\left\{q_{i}(x), p_{j}\left(x^{\prime}\right)\right\}_{\mathrm{DB}} & =\delta_{i j} \delta\left(x-x^{\prime}\right)-\int d x^{\prime \prime} d x^{\prime \prime \prime} \sum_{k . l}\left\{q_{i}(x), \xi_{k}\left(x^{\prime \prime}\right)\right\} C_{k l}\left(x^{\prime \prime}, x^{\prime \prime \prime}\right)\left\{\xi_{l}\left(x^{\prime \prime \prime}\right), p_{j}\left(x^{\prime}\right)\right\} \\
& =: \delta_{i j} \delta\left(x-x^{\prime}\right)-T_{i j}\left(x, x^{\prime}\right)=: K_{i j}\left(x, x^{\prime}\right),
\end{aligned}
$$

where $C_{k l}\left(x, x^{\prime}\right)$ is the inverse of the matrix $\left(\left\{\xi_{i}(x), \xi_{j}\left(x^{\prime}\right)\right\}\right)$ and $x$ denotes space coordinates only. Then smear over the right number of copies of $\mathscr{S}\left(\mathbb{R}^{3}\right)$ and quantize by $\{\cdot, \cdot\}_{\mathrm{DB}} \rightarrow i[\cdot, \cdot]$ to obtain:

$$
[q(F), p(G)]=i(F, G)-i T(F, G)=i K(F, G)
$$

with obvious notation. Because the system is linear, $T(F, G)$ will be a scalar. Hence in the usual manner [10] complexify the test function space to obtain the space $\mathscr{M}$ which comes equipped with the symplectic form

$$
B_{D}\left(F_{1}+i F_{2}, G_{1}+i G_{2}\right):=K\left(F_{1}, G_{2}\right)-K\left(F_{2}, G_{1}\right)=B(F, G)-B^{T}(F, G)
$$

corresponding to the commutation relation of the DB. Here $F=F_{1}+i F_{2}$, $G=G_{1}+i G_{2} \in \mathscr{M}$, and $B$ is the usual symplectic form, $B^{T}$ the extra part. So we can define the field algebra $\mathscr{F}_{D}:=\overline{\Delta\left(\mathscr{M}, B_{D}\right)}$ similar to 2.13 , but this will not be a simple $C^{*}$-algebra because $B_{D}$ is degenerate on $\mathscr{M}$. The degeneracy is due to the fact that $B_{D}$ vanishes on all those $F \in \mathscr{M}$ which correspond to $\left\{\xi_{i}\right\}$. If we call the subspace generated by these $\mathscr{M}^{I I}$, then this should be the degenerate part of $B_{D}$, in which case $\mathscr{M} / \mathscr{M}^{I I}$ will be a nondegenerate symplectic space with respect to the form $\widetilde{B}_{D}$ obtained from $B_{D}$. The step of factoring out $\mathscr{M}^{I I}$ is the step of eliminating the second class constraints from the centre of $\mathscr{F}_{D}$, because

$$
\overline{\Delta\left(\mathscr{M} / \mathscr{M}^{I I}, \widetilde{B}_{D}\right)}=: \mathscr{F}_{0}=\mathscr{F}_{D} / \widetilde{F}_{D} \cdot C^{*}\left(\delta_{\mathscr{H}^{I I}}-\mathbb{1}\right) \text {. }
$$

At this point there are still first class constraints $\mathscr{C} \subset \mathscr{M} / \mathscr{M}^{I I}$ left in the theory, corresponding to $\left\{\chi_{i}\right\}$, and these are removed in the usual $T$-procedure way. Then the final physical algebra is $C^{*}\left(\delta_{\mathbf{p}_{D}}\right) / C^{*}\left(\delta_{\phi_{\delta}}-1\right) C^{*}\left(\delta_{\mathbf{p}_{D}}\right)$ with $\mathbf{p}_{D}:=$ $\left\{F \in \mathscr{M} / \mathscr{M}^{I I} \mid \widetilde{B}_{D}(F, \mathscr{C})=0\right\}$. Now whatever method we follow for removing quantum constraints, we should be able to argue that our resulting physical algebra agrees with or is contained in the physical algebra obtained from heuristic reasoning. So in the examples of Sect. 6 we will compare the physical algebra obtained above with the physical algebra obtained there.

Return now to the general setting of the preceding sections. Whilst it is possible to construct a quantum analog of the DB by alteration of the multiplication laws of the field algebra, we will not attempt that here because we lack suitable examples of nonlinear $C^{*}$-theories for guidance. Instead we propose the method below.

Since $I_{0}$ is compatible, $U_{I_{0}}$ can be interpreted as the largest constraint set which can be put consistently to $\mathbb{1}$ by state condition, and so

$$
\mathscr{D}_{0}:=\left[\mathscr{F} C^{*}\left(U_{I_{0}}-1\right)\right] \cap\left[C^{*}\left(U_{I_{0}}-1\right) \mathscr{F}\right]
$$

is the largest $C^{*}$-algebra which can be consistently set to zero by state condition. 
Henceforth we will interpret "weakly zero" in a quantum context as meaning that an element is annihilated by the Dirac states, the latter being selected by the first class set $U_{I_{0}}$. Now $\mathscr{R}_{0}:=\mathscr{M}_{\mathscr{F}}\left(\mathscr{D}_{0}\right) / \mathscr{D}_{0}$ is not suitable as a physical algebra because we have not yet imposed the second class constraints $U_{I \backslash I_{0}}$. Recall that in step 4 Dirac defined observables as elements having weakly vanishing Poisson brackets with all constraints. We define:

Definition 5.1. The quantum observable algebra is:

$$
\mathcal{O}:=\left\{A \in \mathscr{F} \mid U A U^{-1}-A \in \mathscr{D}_{0} \forall U \in U_{I}\right\} .
$$

That is, $\mathcal{O}$ consists of the "weakly gauge invariant elements," cf. 2.9. Though $U A U^{-1}-A=[U, A] U^{-1}$, we find the form of 5.1. more convenient than the commutator, considering the weakly vanishing $P B$-relations to relate to the infinitesimal generators. Note that the traditional observables $U_{I}^{\prime}$ are in $\mathcal{O}$, and this partly justifies the choice of $\mathcal{O}$. The selection of $\mathcal{O}$ is the step of imposing the second class constraints. The first class constraints can only be imposed on $\mathcal{O}$ if $\mathcal{O} \subset \mathcal{O}_{0}:=\mathscr{M}_{\mathscr{F}}\left(\mathscr{L}_{0}\right)$.

Theorem 5.2. $\mathcal{O} \subset \mathcal{O}_{0}$. If $I_{0}=I$, i.e. all constraints are first class, then $\mathcal{O}=\mathcal{O}_{0}$.

Proof. Since $U_{I_{0}} \subset U_{I}$, we get $\mathcal{O} \subset \mathcal{O}_{0}$ from the definition and from 2.9. If $I=I_{0}$, then 5.1 reduces precisely to 2.9 to give $\mathcal{O}=\mathcal{O}_{0}$.

Hence we can consistently define the maximal physical algebra as:

$$
\mathscr{R}:=\mathcal{O} /\left(\mathcal{O} \cap \mathscr{D}_{0}\right) \text {. }
$$

That it is nontrivial, is clear from the fact that $\mathscr{O} \ni \mathbb{1} \notin \mathscr{D}_{0}$, i.e. $\mathcal{O} \cap \mathscr{D}_{0}$ is a proper ideal of $\mathcal{O}$. This is the actual step of imposing the first class constraints. For consistency, $\mathcal{O}$ should be preserved under the physical automorphisms $\mathscr{E}$.

Theorem 5.4. Given an $\alpha \in$ Aut $\mathscr{F}$ with $\alpha\left(U_{I}\right)=U_{I}$, we find that $\alpha(\mathcal{O}) \subseteq \mathcal{O}$, and hence $\alpha$ defines canonically an automorphism $\alpha^{\prime} \in$ Aut $\mathscr{R}$.

Proof. If $\left(\operatorname{Ad} U_{I}\right) \circ A-A \subset \mathscr{D}_{0}$, then $\alpha\left(\left(\operatorname{Ad} U_{I}\right) \circ A-A\right) \subset \alpha\left(\mathscr{D}_{0}\right)$. The left-hand side is $\left(\operatorname{Ad} U_{I}\right) \circ \alpha(A)-\alpha(A)$ by $\alpha\left(U_{I}\right)=U_{I}$, and the right-hand side is $\alpha\left(\mathscr{D}_{0}\right)=\mathscr{D}_{0}$, which follows from 4.5. So $\left(\operatorname{Ad} U_{I}\right) \circ \alpha(A)-\alpha(A) \subset \mathscr{D}_{0}$ implies $\alpha(A) \in \mathcal{O}$ if $A \in \mathcal{O}$.

So all automorphisms in Ad $U_{I} \cap \mathscr{E}$ are definable as automorphisms on $\mathscr{R}$, and by the two definitions 5.1 and 5.3 are trivial on $\mathscr{R}$, i.e. $\mathscr{R}$ is gauge invariant. It may not be constraint free yet, but if there are $U \in U_{I \backslash I_{0}}$ such that $U \in \mathcal{O}$, then these will end up in the centre of $\mathscr{R}$. At this point we dignify the preceding procedure with a physicality assumption:

Assumption 5.5. Given the constrained system $U_{I} \subset \mathscr{F}_{C}$ as above, all physical information is contained in $\mathscr{R}$ and its set of states.

In practice, $\mathscr{R}$ must be simple if it is to be acceptable as a physical algebra. Hence if it is not, we choose as before a sub $C^{*}$-algebra $\mathbb{U}_{c} \subset \mathbb{U}_{\text {such }}$ that $U_{I}^{\prime} \subset \mathbb{O}_{c}$, invariant with relation to the physical automorphisms $\mathscr{E}$, and such that $\mathscr{R}_{c}:=\mathscr{O}_{c} /\left(\mathscr{O}_{c} \cap \mathscr{D}_{0}\right)$ is simple. This will be the chosen physical algebra. At this point all the constraints are imposed, for those which were in the centre of $\mathscr{R}$ will now be omitted or equal 
to multiples of the identity, since the centre of a simple algebra is trivial. Moreover, the traditional observables $U_{I}^{\prime}$ are in the observable algebra, the physical transformations leave $U_{I}$ and $\mathcal{O}_{c}$ invariant, and the gauge transformations $\operatorname{Ad} U_{I}$ which can be defined on $\mathscr{R}$ are trivial on $\mathscr{R}$. This seems formally satisfactory, especially since the method reduces to the $T$-procedure if $U_{I}$ is first class. It remains to be tested against examples.

As for the state spaces, we see via Dixmier 2.11.6 and 2.11.8 [13] that the states of $\mathscr{R}_{c}$ are in bijection with the states on $\mathscr{O}_{c}$ containing $\mathcal{O}_{c} \cap \mathscr{L}_{0}$ in their kernels, and this bijection respects pure states. Moreover, if $\pi$ and $\xi$ respectively denote the GNS-representation and its cyclic vector of a Dirac state restricted to $\mathcal{C}$, then for all $U \in U_{I} \cap \mathcal{O}$ we have $\pi([A, U]) \xi=0$ for all $A \in \mathcal{O}$.

To conclude this section we summarize our proposed algorithm.

1. Start with a constraint set $U_{I}$ stable under the physical automorphisms $\mathscr{E}$. If we want all gauge automorphisms $\operatorname{Ad} U_{I}$ to be definable on $\mathscr{R}$, enlarge $U_{I}$ to $\operatorname{Ad} U_{I}^{\circ} U_{I}$.

2. Separate $U_{I}$ according to 4.2 into $U_{I}=U_{I_{1}} \cup U_{I_{r}} \cup U_{I_{0}}$.

3. Define $\mathscr{D}_{0}:=\left[\tilde{F} C^{*}\left(U_{I_{0}}-1\right)\right] \cap\left[C^{*}\left(U_{I_{0}}-1\right) \mathscr{F}\right]$ and

$$
\mathcal{O}:=\left\{A \in \mathscr{F} \mid \operatorname{Ad} U_{I} \circ A-A \subset \mathscr{D}_{0}\right\} .
$$

4. Choose an $\mathcal{O}_{c}$ by the requirements above, and define $\mathscr{R}_{c}=\mathscr{C}_{c} /\left(\mathscr{C}_{c} \cap \mathscr{D}_{0}\right)$ as the final physical algebra.

\section{Examples}

\section{A: A Linear Boson Field with Linear Hermitian Constraints}

Heuristically, we know that at least the centre of a given set of constraints should be first class. We prove it in the present context. Let $\{. \|, B\}$ be a symplectic space as before, $\mathscr{F}=\overline{\Delta(\mathscr{M}, B)}$ and define the constraint set as $\|=\delta_{\text {.p. }}$, where $\& \subset . \|$ is the set specifying the constraints, and here will play the role of the index set $I$ of the preceding theory. As for the physical transformations $x: K \mapsto$ Aut $\mathscr{F}$, let these be specified by symplectic transformations $T: K \mapsto S p$. /l. i.e. $\alpha_{k}\left(\delta_{F}\right)=\delta_{T_{k} F}$. Now since $\delta_{-G} \cdot \delta_{F} \cdot \delta_{G}=\delta_{F} \exp i B(G, F)$, we find that Ad $\geqslant /$ will generate multiples of those elements of $\mathscr{U}$ not in its centre, but it leaves the centre invariant. On the other hand, $\alpha_{\boldsymbol{K}}$ will leave $\mathscr{U}$ and Ad $\mathscr{U}_{0} \circ \boldsymbol{U}$ invariant if $T_{K} \mathscr{\delta}=\mathscr{C}_{\text {. }}$. Since $\delta$ "forms an independent basis for $\Delta(\mathscr{M}, B)$, no amount of adjusting of a $\delta_{F}$ by multiples will get it into $\mathscr{U}$ if it is not already there. Hence the only option here for getting a constraint set invariant under the physical transformations. is simply to generate its orbit (cf. Sect. 7). So assume henceforth that $T_{K} 8,=6$, and $\mathrm{Ad}\|\overline{11}=\|$, i.e. we have enlarged $\mathscr{U}$ to the latter set. This is step 1 of the algorithm.

We know from [8] that there is a *-homomorphism

$$
\mathscr{B}:=C^{*}\left(\delta_{H}\right) / C^{*}\left(\delta_{l}-1\right) C^{*}\left(\delta_{H}\right) \rightarrow \widetilde{\Delta\left(H / H_{0}, \widetilde{B}\right)}
$$

for each pair of subspaces $H, L$ of . Il, where $L \subseteq H_{0}$, and the latter denotes the degenerate part of $H$. Hence $/ \mathscr{B}$ is nontrivial if $H / H_{0}$ is nontrivial, in which case

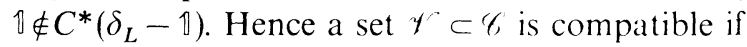


(i) $\mathbf{p}(\mathcal{Y}):=\{F \in \mathscr{U} \mid \mathbb{B}(F, \mathcal{Y})=0\}$ has nontrivial elements not in $\mathcal{Y}$,

(ii) $\exists F, G \in \mathbf{p}(\mathcal{Y})$ such that $B(F, G) \neq 0$, and

(iii) $\mathscr{Y} \subseteq \mathbf{p}(\mathscr{V})_{0}$,

because then $\mathscr{R}=C^{*}\left(\dot{\delta}_{\mathbf{p}(y)}\right) / C^{*}\left(\dot{\delta}_{y}-1\right) C^{*}\left(\dot{\delta}_{\mathbf{p}(y)}\right)$ is nontrivial, and hence by the $T$-procedure $1 \notin C^{*}\left(\delta_{+}-1\right)$. Note that (iii) implies that $\delta_{y}$ is commutative, a fact we already noted earlier in that the elements not in the centre of $\|$ acquired multiples under Ad $\mathscr{U}$, and hence had to be second class. Normally, it seems reasonable to assume that (i) and (ii) will be satisfied for $\mathscr{V}=\mathscr{H}$ in realistic theories, because it means that there is something left in the theory after constraining it. Henceforth we assume (i) and (ii) to hold for $\mathscr{C}$. It also means that (i) and (ii) will be automatically satisfied for all subsets $\mathscr{\mathscr { C }} \subset \mathscr{C}$, because $\mathbf{p}(\mathscr{C}) \subseteq \mathbf{p}(\mathscr{Y})$.

Lemma 6.1. With $\mathscr{C}$ as above, a subset $\mathscr{Y} \subset \mathscr{C}$ is compatible iff $\mathscr{Y} \subset \mathbf{p}(\mathscr{Y})_{0}$.

Proof. By (iii) above we have already that $\mathscr{Y}^{\wedge} \subset \mathbf{p}\left(\mathscr{Y}_{0}\right)_{0}$ implies $\mathscr{Y}^{\wedge}$ is compatible. We show the converse. Assume $\mathscr{V} \not \mathbf{p}(\mathscr{Y})_{0}$, i.e. $\exists F, G \in \mathscr{Y}$ such that $B(F, G) \neq 0$. Then $\left[\delta_{F}-1, \delta_{G}-1\right]=\delta_{F+G} 2 \sin B(F, G) \in C^{*}\left(\delta_{y}-1\right)$, hence since $B(F, G) \neq 0$, the unitary $\delta_{F+G} \in C^{*}\left(\delta_{\gamma}-1\right)$, and so $\mathscr{Y}$ is not compatible.

Corollary 6.2. Two self compatible subsets $\mathscr{Y}, \mathscr{H} \subset \mathscr{C}$ are mutually compatible iff $B(\mathscr{V}, \mathscr{W})=0$.

Recall that the first class constraint set $I_{0} \subset \mathscr{C}$ consists of all the elements which are compatible with all self compatible sets.

Corollary 6.3. $I_{0}=\mathscr{C}_{0}$, the degenerate part of $\mathscr{C}$ under $B$ is the same as its first class part.

Proof. Each $F \in \mathscr{C}$ is self compatible, because from $B(F, F)=0$, Lemma 6.1 holds. Hence $I_{0} \subseteq\{F \in \mathscr{C} \mid B(F, G)=0 \forall G \in \mathscr{C}\}=: \mathscr{C}_{0}$. Reverse inclusion follows from the linearity of $B$.

This establishes the heuristic result, because $\delta_{b_{0}}$ is the centre of $\%$, and concludes step 2 of our algorithm. So

and

$$
\begin{aligned}
\mathscr{L}_{0} & =\left[\Delta(\mathscr{H}, B) C^{*}\left(\delta_{e_{0}}-1\right)\right] \cap\left[C^{*}\left(\delta_{\varphi_{0}}-\hat{1}\right) \Delta(\mathscr{H}, B)\right] \\
& =\left[\delta_{\mathscr{H}}\left(\delta_{\mathscr{R}_{0}}-1\right)\right] \cap\left[\left(\delta_{\mathscr{R}_{0}}-1\right) \delta_{\mathscr{M}}\right],
\end{aligned}
$$

$$
\mathbb{C}:=\left\{A \in \widetilde{F} \mid \delta_{F} A \delta_{-F}-A \in \mathscr{L}{ }_{0} \forall F \in \mathscr{C}\right\} .
$$

So $\delta_{G} \in \mathcal{O}$ if $\delta_{F} \delta_{G} \delta_{-F}-\delta_{G}=\delta_{G} \exp i B(G, F)-\delta_{G}=(\exp i B(G, F)-1) \delta_{G} \in \mathscr{Z}_{0}$. Hence since $\mathscr{D}_{0}$ is a $C^{*}$-algebra, either $B(G, F)=0 \forall F \in \mathscr{C}$, or $\delta_{G} \in \mathscr{L}_{0}$, but the latter alternative cannot be, because it implies that $1 \in \mathscr{Z}_{0}$, in contradiction with $\mathscr{C}_{0}$ being first class. Thus $\delta_{G} \in \mathcal{O}$ iff $B(G, \mathscr{C})=0$. Thus as before, we define the physical subspace $\mathbf{p}(\mathscr{C})=\{F \in \mathscr{M} \mid B(F, \mathscr{C})=0\}$ with respect to $\mathscr{C}$, and choose $C_{c}=C^{*}\left(\dot{\delta}_{\mathbf{p}(\mathscr{G})}\right) \subset \mathcal{C}$, then $\mathscr{U}^{\prime}=\mathcal{O}_{c}$, and since $T_{k}$ is symplectic and $T_{K} \mathscr{C}=\mathscr{C}$. then also $T_{K} \mathbf{p}(\mathscr{C})=\mathbf{p}(\mathscr{C})$, and so $x_{K}\left(C_{c}\right)=C_{c}$. Note that ${ }^{\prime} f_{0}^{\prime}=\left(f \cap \mathbf{p}(\ell)\right.$. By theorem 3.2 of $[8]$. $C_{0} \cap C_{c}=$ $C^{*}\left(\delta_{\varepsilon_{0}}-1\right) C^{*}\left(\delta_{p(s)}\right)$, and hence

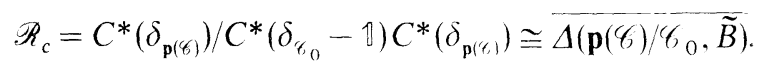

Here we also took the additional step of assuming $\mathbf{p}\left(\phi_{6}\right)_{0}=\mathscr{6}_{0}$. or if it is not, to 
also factor out the remaining elements in $\mathbf{p}(\mathscr{C})_{0}$ to ensure that $\mathscr{R}_{c}$ is simple. This concludes the application of the proposed algorithm to the present example. We now return to the problem of its justification, by comparing the physical algebra just obtained with the one obtained early in Sect. 5 on employing the Dirac bracket notion. The earlier physical algebra $\mathscr{R}_{D}:=C^{*}\left(\delta_{\mathbf{p}_{D}}\right) / C^{*}\left(\delta_{\zeta_{0}}-1\right) C^{*}\left(\delta_{\mathbf{p}_{D}}\right)$ was obtained from two successive factorizations, but it would have been the same to do it in one step. Define first $\mathbf{p}_{D}\left(\mathscr{C}_{0}\right):=\left\{F \in \mathscr{M} \mid B_{D}\left(F, \mathscr{C}_{0}\right)=0\right\} \supset \mathscr{M}^{I I}=\mathscr{C}_{r}$, and then

$$
\begin{aligned}
\mathscr{R}_{D} & =C^{*}\left(\delta_{\mathbf{p}_{D}\left(\mathscr{C}_{0}\right)}\right) / C^{*}\left(\delta_{\mathbf{p}_{D}\left(\mathscr{C}_{0}\right)_{0}}-1\right) C^{*}\left(\delta_{\mathbf{p}_{D}\left(\mathscr{C}_{0}\right)}\right), \\
& \cong \overline{\Delta\left(\mathbf{p}_{D}\left(\mathscr{C}_{0}\right) / \mathbf{p}_{D}\left(\mathscr{C}_{0}\right)_{0}, \widetilde{B}_{D}\right)}
\end{aligned}
$$

where likewise we took the liberty of factoring out the additional elements of $\mathbf{p}_{D}\left(\mathscr{C}_{0}\right)_{0}$ not in $\mathscr{C}_{0}$, in order to make $\mathscr{R}_{D}$ simple. So now we need to compare $\mathscr{R}_{D}$ with $\mathscr{R}_{c}$, which means that it is the relation between the symplectic spaces $\left\{\mathbf{p}(\mathscr{C}) / \mathbf{p}(\mathscr{C})_{0}, \widetilde{B}\right\}$ and $\left\{\mathbf{p}_{D}\left(\mathscr{C}_{0}\right) / \mathbf{p}_{D}\left(\mathscr{C}_{0}\right)_{0}, \widetilde{B}_{D}\right\}$ which should be looked at. At this point it is necessary to make an assumption on the behaviour of $B^{T}$. Recall that $B^{T}$ comes from the term $\int d x d x^{\prime} \sum_{k, l}\left\{;, \xi_{k}(x)\right\} C_{k l}\left(x, x^{\prime}\right)\left\{\xi_{l}\left(x^{\prime}\right), \cdot\right\}$, where the $\xi$ are second class constraints. Hence we expect the following behaviour from $B^{T}: B^{T}\left(\mathscr{C}_{0}, F\right)=0$ $\forall F \in \mathscr{M}$, because in this context the first class constraints commute with all the other constraints, and $B^{T}(\mathbf{p}(\mathscr{C}), F)=0 \forall F \in \mathscr{M}$ for a similar reason for the physical space. Since $\mathscr{C}_{0} \subset \mathbf{p}(\mathscr{C})$, the first relation is contained in the second. Moreover, $B_{D}$ is set up to eliminate second class constraints: $B_{D}\left(\mathscr{C} \backslash \mathscr{C}_{0}, F\right)=0 \forall F \in \mathscr{M}$. Henceforth we assume the behaviour above of $B^{T}$. Then $B(F, G)=B_{D}(F, G) \forall F \in \mathbf{p}(\mathscr{C}), G \in \mathscr{M}$. Hence $\mathbf{p}(\mathscr{C}) \subset \mathbf{p}_{D}\left(\mathscr{C}_{0}\right)$ and $\mathbf{p}_{D}\left(\mathscr{C}_{0}\right)_{0} \cap \mathbf{p}(\mathscr{C}) \subseteq \mathbf{p}(\mathscr{C})_{0}$, and on $\mathbf{p}(\mathscr{C})$ the forms $B$ and $B_{D}$ are equal. So we see that $\mathscr{R}_{c}$ is a factor algebra of a subalgebra of $\mathscr{R}_{D}$, and hence the algorithm we have proposed appears to be stricter than that suggested by the Dirac algorithm.

Next we wish to apply the theory of linear boson fields above to a real example which occurred in the literature; the anomalous chiral Schwinger model, worked out in two dimensions by Rajaraman [14]. At the point before construction of the $\mathrm{DB}$ one has (in his notation) three canonical pairs with equal time $P B$-relations:

$$
\left\{A_{0}(x), \pi_{0}(y)\right\}=\left\{A_{1}(x), E(y)\right\}=\{\varphi(x), \pi(y)\}=\delta(x-y)
$$

with constraints:

$$
\begin{aligned}
\Omega_{1}(x) & :=\pi_{0}(x) \approx 0 \\
\Omega_{2}(x) & :=E^{\prime}(x)+e\left(\pi(x)+\varphi^{\prime}(x)\right)+e^{2} A_{1}(x)+e^{2}(a-1) A_{0}(x) \\
\left\{\Omega_{1}(x), \Omega_{2}(y)\right\} & =e^{2}(a-1) \delta(x-y),
\end{aligned}
$$

and the Hamiltonian which preserves the constraint set is:

$$
\begin{aligned}
H= & \int d x\left\{\frac{1}{2} \pi^{2}+\frac{1}{2} \varphi^{\prime 2}+\frac{1}{2} E^{2}-E^{\prime} A_{0}-e\left(\pi+\varphi^{\prime}\right)\left(A_{0}-A_{1}\right)+\frac{1}{2} e^{2}\left(A_{0}-A_{1}\right)^{2}\right. \\
& \left.-\frac{1}{2} a e^{2}\left(A_{0}^{2}-A_{1}^{2}\right)+\pi_{0}\left[A_{1}^{\prime}-(a-1)^{-1} E\right]\right\} .
\end{aligned}
$$

The situation is linear, and hence the preceding theory is applicable. Since the time evolutions preserve the constraints, we will not further consider these, since it is only a matter of writing down the explicit time evolution, and by construction we 
know that this will be symplectic (cf. [15] for an example of this). Smear the relations in the free space variable over $\mathscr{S}(\mathbb{R})$, and so to quantize we complexify $\mathscr{S}(\mathbb{R})$ and take three copies of it for the symplectic space $\mathscr{M}$. The correspondence will be (with notation $F_{l}=\mathfrak{R} F_{l}+i \mathfrak{I} F_{l}$ for real and imaginary parts).

$$
F=\left(F_{1}, F_{2}, F_{3}\right) \leftrightarrow\left(\pi_{0}\left(\mathfrak{R} F_{1}\right)+A_{0}\left(\mathfrak{I} F_{1}\right), E\left(\Re F_{2}\right)+A_{1}\left(\mathfrak{I} F_{2}\right), \pi\left(\mathfrak{R} F_{3}\right)+\varphi\left(\mathfrak{I} F_{3}\right)\right),
$$

where $F_{l} \in \mathscr{S}(\mathbb{R})+i \mathscr{S}(\mathbb{R}), l=1,2,3$. The symplectic form corresponding to the CCR is:

$$
B(F, G)=\int d x \sum_{i=1}^{3}\left(\mathfrak{R} F_{i} \mathfrak{J} G_{i}-\mathfrak{J} F_{i} \mathfrak{R} G_{i}\right) .
$$

On utilizing the correspondence above, we get for $\Omega_{1}, \Omega_{2}$ respectively the constraint spaces:

$$
\begin{aligned}
\mathscr{C}_{1} & :=\{F \in \mathscr{M} \mid F=(f+i 0,0,0), f \in \mathscr{P}(\mathbb{R})\} \text { and } \\
\mathscr{C}_{2}: & =\left\{F \in \mathscr{M} \mid F=\left(i e^{2}(a-1) f,-f^{\prime}+i e^{2} f, e f-i e f^{\prime}\right), f \in \mathscr{S}(\mathbb{R})\right\} \\
& =\left\{F \in \mathscr{M} \mid F=\left(i e^{2}(a-1),-\partial+i e^{2}, e-i e \partial\right) f, f \in \mathscr{S}(\mathbb{R})\right\} .
\end{aligned}
$$

Then $\mathscr{U}=\left(\operatorname{Ad} \delta_{\mathscr{C}}\right) \circ \delta_{\mathscr{C}}, \mathscr{C}=\mathscr{C}_{1} \cup \mathscr{C}_{2}$. So from the noncommutativity of the first components, everything in $\delta_{\mathscr{E}}$ get multiples of themselves, and so is second class. It can be seen at a glance from $\mathscr{C}_{1}$ and $\mathscr{C}_{2}$ that there is nothing first class, i.e. $\mathbf{p}(\mathscr{C}) \cap \mathscr{C}=\{0\}$. So in this case $\mathscr{R}_{c}=\overline{\Delta\left(\mathbf{p}(\mathscr{C}) / \mathbf{p}(\mathscr{C})_{0}, \widetilde{B}\right)}$ and $\mathscr{O}_{c}=C^{*}\left(\delta_{\mathbf{p}(\mathscr{C})}\right), \mathscr{D}_{0}=\{0\}$. Now $\mathbf{p}(\mathscr{C})=\{F \in \mathscr{M} \mid B(F, \mathscr{C})=0\}$. So for $F \in \mathbf{p}(\mathscr{C})$,

$$
\begin{aligned}
B(F, C)= & \int d x\left\{\left(\Re F_{1}\right) e^{2}(a-1) h-\left(\mathfrak{I} F_{1}\right) g+\left(\Re F_{2}\right) e^{2} h+\left(\mathfrak{J} F_{2}\right) h^{\prime}-\left(\mathfrak{R} F_{3}\right) e h^{\prime}\right. \\
& \left.-\left(\mathfrak{J} F_{3}\right) e h\right\}=0 \quad \forall g, h \in \mathscr{S}(\mathbb{R}) .
\end{aligned}
$$

Hence $\mathfrak{I} F_{1}=0$, and $e^{2}(a-1) \mathfrak{R} F_{1}+e^{2} \mathfrak{R} F_{2}-\partial \mathfrak{I} F_{2}+e \partial \mathfrak{R} F_{3}-e \mathfrak{I} F_{3}=0$. Use this to eliminate $\mathfrak{R} F_{1}$, hence:

$\mathbf{p}(\mathscr{C})=\left\{F \in \mathscr{M} \mid F=\left(e^{-2}(a-1)^{-1}\left[-e^{2} \mathfrak{R} F_{2}+\partial \mathfrak{J} F_{2}-e \partial \mathfrak{R} F_{3}+e \mathfrak{I} F_{3}\right]+i 0, F_{2}, F_{3}\right)\right\}$

Also, $\mathbf{p}(\mathscr{C})_{0}=\{0\}$, because the first components in $\mathbf{p}(\mathscr{C})$ have no imaginary parts, hence do not contribute to $B(F, G), F, G \in \mathbf{p}(\mathscr{C})$, whilst other components can still be arbitrary in $\mathscr{S}(\mathbb{R})+i \mathscr{S}(\mathbb{R})$. Hence $\mathscr{R}_{c}=\overline{\Delta(\mathbf{p}(\mathscr{C}), B)}$, i.e. the simple commutator of the constraints.

\section{B. Linear Boson Fields with Quadratic Constraints}

Consider the heuristic situation where a canonical pair $p, q$ is embedded in a larger system, and the constraints given are $p, q$ and $p q$. Then $p, q$ are second class, but since $[p, p q]=i p \approx 0$ and $[q, p q]=-i q \approx 0$, the constraint $p q$ is first class. We intend to model this situation in the rigorous framework, to see whether the abstract algorithm agrees with this classification of constraints. The tools for dealing with quadratic constraints were developed in [8], and was touched on in the conclusion of Sect. 2.

Let $\mathscr{F}=\overline{\Delta(\mathscr{M}, B)}$ where $\mathscr{M}=\mathscr{S}(\mathbb{R})+i \mathscr{P}(\mathbb{R}), B(F, G)=\int(\mathfrak{R} F \cdot \mathfrak{I} F-\mathfrak{I} F \cdot \mathfrak{R} G)$. As before, $F \in \mathscr{M}$ corresponds to $p(\mathfrak{R} F)+q(\mathfrak{I} F)$. Let $J \subset \mathbb{R}$ be a closed bounded interval, 
and define $\mathscr{C}_{1}:=\{F \in \mathscr{M} \mid \operatorname{supp} F \subseteq J\}$, and this will correspond to a boson system on $J$. To define the $p q$ constraint cf. [8], we need to define the automorphism on $\mathscr{F}$, Ad exp $i \lambda \int(p q f), \lambda \in \mathbb{R}, f \in \mathscr{S}(\mathbb{R})$. Hermitian reordering is not necessary, because $p q, q p$ and $\frac{1}{2}(p q+q p)$ all have identical commutators with $q$ and $p$. Now

$$
\delta_{F} \leftrightarrow W(F)=\exp i(p(\Re F)+q(\Im F)) .
$$

We find $\left[i \lambda(p q)(f), p\left(F_{1}\right)+q\left(F_{2}\right)\right]=\lambda\left(p\left(f F_{1}\right)-q\left(f F_{2}\right)\right)$, and hence

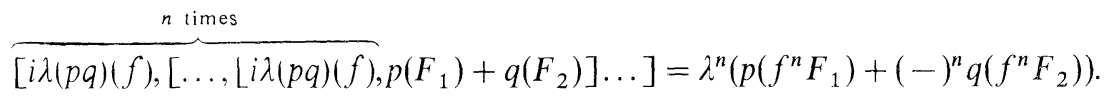

So

$$
\begin{aligned}
(\operatorname{Ad} \exp i \lambda(p q)(f))\left(p\left(F_{1}\right)+q\left(F_{2}\right)\right) & =(\exp \operatorname{ad} i \lambda(p q)(f))\left(p\left(F_{1}\right)+q\left(F_{2}\right)\right) \\
& =p\left(e^{\lambda f} F_{1}\right)+q\left(e^{-\lambda f} F_{2}\right) .
\end{aligned}
$$

On employment of $(\operatorname{Ad} U) A^{n}=((\operatorname{Ad} U) A)^{n}$ and a power series expansion:

$$
(\operatorname{Ad} \exp i \lambda(p q)(f)) \exp i\left(p\left(F_{1}\right)+q\left(F_{2}\right)\right)=\exp i\left(p\left(e^{\lambda f} F_{1}\right)+q\left(e^{-\lambda f} F_{2}\right)\right) .
$$

Hence in the rigorous arena, this automorphism corresponds to a transformation on $\mathscr{M}$ given by:

$$
T_{\lambda f} F=e^{\lambda f} \mathfrak{R} F+i e^{-\lambda f} \mathfrak{I} F \in \mathscr{M}, \quad \lambda \in \mathbb{R}, \quad f \in \mathscr{S}(\mathbb{R}) .
$$

By inspection we see that $T_{\lambda f}$ is symplectic, hence defines an automorphism group on $\mathscr{F}$ by $\alpha_{\lambda f}\left(\delta_{F}\right)=\delta_{T, f}$. As before in [8], we take the inductive limit of the $C^{*}$-algebras in the multiplier algebras of the crossed products, to get $\mathscr{F}_{e}$ and the usual outer constraint structure, i.e. elements $U_{\lambda f} \in\left(\mathscr{F}_{e}\right)_{u}$ such that $\alpha_{\lambda f}=\operatorname{Ad} U_{\lambda f}$, and the $U_{\lambda f}$ are identified with the $\exp i \lambda(p q)(f)$. Hence $\mathscr{C}_{2}:=\{f \in \mathscr{S}(\mathbb{R}) \mid \operatorname{supp} f \subseteq J\}$ will be the index set of the $U_{\lambda f}$ constraints, the full index set being $I=\mathscr{C}_{1} \cup \mathscr{C}_{2}$. Now $\operatorname{Ad} U_{\mathscr{C}_{1}}{ }^{\circ} U_{\mathscr{C}_{1}}$ just generates the usual multiples on $U_{\mathscr{C}_{1}}=\delta_{\mathscr{C}_{1}}, \operatorname{Ad} U_{\mathscr{t}_{2}}{ }^{\circ} U_{\mathscr{\ell}_{1}}=$ $\alpha_{\mathscr{q}_{2}}\left(U_{\mathscr{q}_{1}}\right)=U_{\mathscr{q}_{1}}$, and hence will preserve this set. $\operatorname{Ad} U_{\mathscr{q}_{2}}{ }^{\circ} U_{\mathscr{V}_{2}}$ is preserved by the group property, and $\operatorname{Ad} U_{\mathscr{Q}_{1}} \circ U_{\mathscr{C}_{2}}=\left\{U_{\lambda f} \delta_{T_{-\lambda f} F} \delta_{-F} \mid f \in \mathscr{C}_{2}, F \in \mathscr{C}_{1}\right\}$, which is not in $\mathscr{F}$. Clearly due to the multiples, $\mathscr{C}_{1}$ is not a compatible set, although it does contain

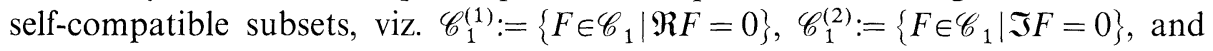
these are indeed representative, in that any index set which is compatible with both these sets, will be compatible with all self-compatible subsets of $\mathscr{C}_{1}$. So first, we want to show that $\mathscr{C}_{2}$ is compatible with both $\mathscr{C}_{1}^{(1)}$ and $\mathscr{C}_{1}^{(2)}$, which entails of course that $\mathscr{C}_{2}$ is self-compatible. According to Theorem 2.15, for proving that a self-compatible set $\mathscr{V} \subset \mathscr{C}_{1}$ is compatible with $\mathscr{C}_{2}$, it suffices to show that there exists an $\alpha$-invariant state on $\mathscr{F}$ which also vanishes on $\left\{\delta_{r}-1\right\}$. This is very easy when $\mathscr{V}$ is a linear space invariant under $T_{\mathscr{C}_{2}}$, since the required state $\omega$ is then simply defined by $\omega\left(\delta_{\mathscr{V}}\right)=1$, and $\omega\left(\delta_{F}\right)=0$ when $F \notin \mathscr{V}$. Hence it suffices to show here that the linear spaces $\mathscr{C}_{1}^{(1)}$ and $\mathscr{C}_{1}^{(2)}$ are $T_{\mathscr{C}_{2}}$-invariant, and this is almost trivial to see, because $T_{\lambda f}$ does not mix the real and imaginary parts of $\mathscr{M}$, and so $T_{\mathscr{C}_{2}} \mathscr{C}_{1}^{(1)}=\mathscr{C}_{1}^{(1)}, T_{\mathscr{C}_{2}} \mathscr{C}_{1}^{(2)}=\mathscr{C}_{1}^{(2)}$, i.e. $\mathscr{C}_{2}$ is compatible with both $\mathscr{C}_{1}^{(1)}$ and $\mathscr{C}_{1}^{(2)}$, hence with $\mathscr{C}_{1}$. Finally, we want to show that $\operatorname{Ad} U_{\mathscr{C}_{1}}{ }^{\circ} U_{\mathscr{C}_{2}}$ has no first class part, and that all its self-compatible subsets are compatible with $\mathscr{C}_{2}$. Towards this, 
note first that since $\operatorname{Ad} U_{\mathscr{C}_{1}}$ consists of automorphisms, they therefore preserve the identity and hence map compatible sets to compatible sets. So the compatibility structures of $\mathscr{C}_{1}$ are carried over identically. Moreover, $C^{*}\left(U_{\mathscr{C}_{2}} \cup \operatorname{Ad} U_{\mathscr{C}_{2}}{ }^{\circ} U_{\mathscr{C}_{1}^{(i)}}-\mathbb{1}\right)=$ $C^{*}\left(U_{\mathscr{C}_{2}} \cup U_{\mathscr{C}_{1}^{(i)}}-\mathbb{1}\right)$, hence we have already proven that $\mathscr{C}_{2}$ is compatible with all self-compatible subsets of $\operatorname{Ad} U_{\mathscr{C}_{2}} \circ U_{\mathscr{C}_{1}}$, the latter of which consists of commutative subsets. Finally, note that the centre of $\delta_{\mathscr{C}_{1}}$ is 1 . This concludes the proof that $\mathscr{C}_{2}$ is the first class part of $\mathscr{C}$, a result fully in agreement with the heuristic one.

So now $\mathscr{D}_{0}=\left[\mathscr{F}_{e} C^{*}\left(U_{\mathscr{C}_{2}}-\mathbb{1}\right)\right] \cap\left[C^{*}\left(U_{\mathscr{C}_{2}}-\mathbb{1}\right) \mathscr{F}_{e}\right] \cap \mathscr{F}$, and by the formal theory we get $A \in \mathscr{D}_{0}$ iff $\omega\left(A^{*} A\right)=0=\omega\left(A A^{*}\right) \forall \omega \in \Xi^{\alpha}(\mathscr{F})$ where the latter denotes the $\alpha$-invariant states on $\mathscr{F} . \mathscr{O}=\left\{A \in \mathscr{F} \mid \operatorname{Ad} U_{I}^{\circ} A-A \subset \mathscr{D}_{0}\right\}$, and clearly by the previous example $\delta_{F} \in \mathcal{O} \Rightarrow F \in \mathbf{p}\left(\mathscr{C}_{1}\right)=\{F \in \mathscr{M} \mid \operatorname{supp} F \subset \mathbb{R} \backslash J\}$. Then for $\mathscr{C}_{2}$ we have $\delta_{F} \in \mathcal{O}$ iff $\delta_{T_{\lambda, F} F}-\delta_{F} \in \mathscr{D}_{0} \forall f \in \mathscr{C}_{2}$ iff $\omega\left(21-\delta_{F} \cdot \delta_{-T_{\lambda, f} F}-\delta_{T_{\lambda, f} F} \delta_{-F}\right)=0 \forall \omega \in \mathbb{S}^{\alpha}(\mathscr{F})$ iff

$$
2-\omega\left(\delta_{F-T_{\lambda f} F}\right) \exp \frac{-i}{2} B\left(F, T_{\lambda f} F\right)-\omega\left(\delta_{T_{\lambda f} F-F}\right) \exp \frac{-i}{2} B\left(T_{\lambda f} F, F\right)=0 .
$$

Now if $F \in \mathbf{p}\left(\mathscr{C}_{1}\right), f \in \mathscr{C}_{2}$, then their supports are disjunct so we see by inspection that $B\left(F, T_{\lambda f} F\right)=0$, and that $T_{\lambda f} F-F=0$, hence $\omega\left(\delta_{T_{\lambda f} F-F}\right)=1$. So this relation is automatically satisfied, and hence $\delta_{F} \in \mathcal{O}$ iff $F \in \mathbf{p}\left(\mathscr{C}_{1}\right)$. So we could choose $\mathcal{O}_{c}=C^{*}\left(\delta_{\mathbf{p}\left(\mathscr{C}_{1}\right)}\right)$, and since $B$ is nondegenerate on $\mathbf{p}\left(\mathscr{C}_{1}\right), \mathscr{R}_{c}=\mathcal{O}_{c}=\overline{\Delta\left(\mathbf{p}\left(\mathscr{C}_{1}\right), B\right)}$. This concludes the present section.

\section{Adjusting the Dynamics}

Recall that steps 2 and 3 of the Dirac procedure consists of the adjustment of the Hamiltonian by combinations of constraints in order to minimize the orbit of the constraint set under time evolutions. This minimal orbit is then taken as the full constraint set to be imposed. In the kinematics problem set up above, we assumed that the equivalent of this procedure has already been carried out in the $C^{*}$-theory. We now consider this equivalent procedure.

There is no Hamiltonian in the $C^{*}$-picture; it only exists for certain representations. Nevertheless, we do have a one-parameter group of time automorphisms to describe the dynamics, and so the problem of steps 2 and 3 of the Dirac procedure will translate in this picture to the problem of how to adjust this automorphism group by objects constructed from the constraints in such a way as to minimize the orbit of the constraint set. If one simply enlarged the constraint set to its full orbit under the unaltered dynamics group, the wrong physics is obtained as can be seen from simple examples. It was possible to construct several reasonable procedures in the $C^{*}$-picture which behaved in an analogous way to the classical Dirac procedure, but we cannot claim that any of these solved the problem, for the following reasons.

As the multipliers $\alpha_{i}$ of the constraints in $H_{P}=H+\alpha_{i} \Omega_{i}$ are phase space functionals, and those of the second class constraints get fixed, it is often the case that $H_{P}$ is a higher than quadratic polynomial in $p$ and $q$. Hence the dynamics often do not conserve the linear part of the theory. In fact this is found to be very useful in physics for introducing interaction into a linear theory. Now in a $C^{*}$-setting 
we know that both the CCR and CAR $C^{*}$-algebras can only model linear field theories, although there are constructions for obtaining nonlinear objects from these. This means that whatever method we use for adjustment of the quantum dynamics by constraints, it should refuse to work in the $C^{*}$-algebras of linear theories until these have been enlarged to contain sufficient nonlinear objects. So the right method for dynamics adjustment should be algebra dependent. As it is still one of the main outstanding problems of mathematical physics to construct a $C^{*}$-algebra which describes a nonlinear field theory (higher than quadratic), it is not possible to specify precisely how such a method should be algebra dependent in a $C^{*}$-context, and so the dynamics adjustment problem remains ill defined. That is, we may evolve a method of dynamics adjustment in the general category of $C^{*}$-algebras which has some of the Dirac procedure's characteristics, but as there are no suitable examples in the $C^{*}$-framework to apply it to, there is no way to decide if such a method is acceptable from the point of view of physics or not. We must wait for the $C^{*}$-theory of nonlinear field theories to evolve before we can sensibly approach the dynamics adjustment problem.

\section{Conclusions}

In this paper, we have proposed an algorithm for dealing with systems with second class constraints in a quantum framework. When tested on examples, it behaved in a proper fashion. At this point its usefulness only extends as far as the $C^{*}$-algebraic framework is applicable to quantum theory, but it does suggest in its structure analogous methods in other formulations of quantum physics. An aspect of the algorithm which still needs further development is the first step, adjusting the physical automorphism group and constraint set in to a stable system, but that has not been pursued due to the lack of a suitable example of a nonlinear $C^{*}$-field theory. Further questions arise from the algorithm, e.g. what conditions should a physical automorphism satisfy on $\mathscr{F}$ in order to be inner on $\mathscr{R}_{c}$, when are indefinite inner product representations required etc. We leave such questions for future investigations.

\section{References}

1. Dirac, P. A. M.: Lectures in quantum mechanics. New York: Belfer Graduate School of Science, Yeshiva University 1964; Sudarshan, E. C. G., Mukunda, N.: Classical dynamics: A modern perspective. New York: John Wiley 1974; Sundermeyer, K.: Constrained dynamics. Lecture Notes in Physics Vol. 169. Berlin, Heidelberg New York: Springer 1982

2. Gotay, M. J., Nester, J. M., Hinds, G.: Presymplectic manifolds and the Dirac-Bergman theory of constraints. J. Math. Phys. 19, 2388-2399 (1978)

3. Dirac, P. A. M.: Generalised Hamiltonian dynamics. Can. J. Math. 2, 129-148 (1950); Faddeev, L. D.: The Feynman integral for singular Lagrangians. Theor. Math. Phys. 1, 1-12 (1970)

4. Abraham, R., Marsden, J.: Foundations of mechanics. New York: Benjamin 1967; Chernoff, P. R.: Mathematical obstructions to quantization. Had. J. 4, 879-898 (1981); Kálnay, A. J.: On certain intriguing physical, mathematical and logical aspects concerning quantizatıon. Had. J. 4, 1127-1165 (1981)

5. Gotay, M. J., Sniatycki, J.: On the quantization of presymplectic dynamical systems via coisotropic imbeddings. Commun. Math. Phys. 82, 377-389 (1981) 
6. Grundling, H. B. G. S., Hurst, C. A.: Algebraic quantization of systems with a gauge degeneracy. Commun. Math. Phys. 98, 369-390 (1985)

7. Grundling, H., Hurst, C. A.: Algebraic structures of degenerate systems and the indefinite metric. J. Math. Phys. 28, 559-572 (1987)

8. Grundling, H.: Systems with outer constraints. Gupta-Bleuler electromagnetism as an algebraic field theory. Commun. Math. Phys. 114, 69 (1988)

9. Shanmugadhasan, S.: Canonical formalism for degenerate Lagrangians. J. Math. Phys. 14, 677-687 (1975)

10. Emch, G. G : Algebraic methods in statistical mechanics and quantum field theory. New York: John Wiley 1972

11. Manuceau, J.: $C^{*}$-algebre de relations de commutation. Ann. Inst. Henri Poincaré 8, 139-161 (1968)

12. Blackadar, B.: K-theory for operator algebras. Berlin, Heidelberg, New York: Springer 1986

13. Dixmier, J.: $C^{*}$-algebras. Amsterdam: North Holland 1977

14. Rajaraman, R: Hamiltonian formulation of the anomalous chiral Schwinger model. Phys. Lett. 154B, 305-309 (1985)

15. Grundling, H.: Algebraic structures of degenerate systems. Adelaide University, Ph.D. thesis 1986

16. Slawny, J.: On factor representations and the $C^{*}$-algebra of the canonical commutation relations. Commun. Math. Phys. 24, 151-170 (1972)

17. Faddeev, L. D.: Operator anomaly for the Gauss law. Phys. Lett. 145B, 81-84 (1984)

Communicated by H. Araki

Received March 7, 1988 
